\title{
Modeling of Radiated Noise Passing Characteristic for Ship in Different Marine Environments
}

\author{
Liang Zhang ${ }^{1}$ and Chunxia Meng, ${ }^{2, *}$ \\ ${ }^{1}$ Dalian Institute of Measurement and Control Technology, China \\ ${ }^{2}$ Science and Technology on Underwater Test and Control Laboratory, China \\ ${ }^{*}$ Corresponding author
}

\begin{abstract}
The ship radiated noise is used as information sources of passive detection and classification, at the same time, the realistic simulation of ship radiated noise signals is an effective way to deceive the passive acoustic detection equipment. In this paper, we analyze a large amount of near field radiation noise data under the same conditions for a certain type of ship, and obtain the statistical characteristics of the radiated noise spectrum a set of random numbers satisfying this statistical feature was generates using a probability density function to describe the spectrum of the underwater noise source. Use it as an input signal to the filter, an ocean channel filter with arbitrary acoustic velocity profile was established on ray acoustics, and the Fourier synthesis algorithm was used to reconstruct the passing characteristics of the radiated noise signal received by the hydrophone at any depth in a typical shallow sea and deep sea. Finally, the effectiveness of the reconstructed radiated noise waveform is analyzed. The results show that the correlation between the reconstructed signal and the measured ship signal in the frequency domain is greater than 0.8 . The modeling method in this paper is very important for the analysis of underwater acoustic signals in the laboratory environment.
\end{abstract}

Keywords-marine environment; radiated noise for ship; passing characteristic

\section{INTRODUCTION}

When a ship is sailing or operating, the propeller and various kinds of machinery are working. The vibrations generated by them radiate sound waves into the water through the hull. This forms the radiation noise of the ship. Ship radiated noise is the combined effect of numerous noise sources. These sources include propellers, rotating and reciprocating machines, and various types of pumps. Their mechanisms for generating noise are different. Therefore, the spectral shape of radiated noise is also more complicated [1]. In general, there are two basic types of noise spectrum. One is continuous spectrum. Its noise level is a continuous function of frequency, and the other is single frequency noise. Its spectral shape is line spectrum [2]. For ship radiated noise, in a large frequency range, the noise is a mixture of the above two types of noise. The spectral shape is the superposition of the line spectrum and the continuous spectrum. When the sailing conditions are constant, due to the mechanical equipment itself, the intensity of the line spectrum of ship radiation noise and the slope of the continuous spectrum will change slightly [3]. Therefore, the results of a single measurement at sea can't represent the inherent characteristics of ship radiated noise. When the ship is sailing in the ocean, it is affected by the ocean filter, and amplitude and phase of the received signal of the hydrophone are distorted. At this time, the inherent characteristics of ship radiated noise become insignificant. In view of this, based on the statistical characteristics of spectrum obtained from the near field measured data, this paper carries out the modeling research on the ship's radiated noise passing characteristics in different marine environments, and uses the correlation analysis method to evaluate the effectiveness of the reconstructed signal.

\section{SHIP'S RADIATED NOISE SPECTRUM CHARACTERISTICS BASED ON STATISTICAL ANALYSIS}

Usually, the radiated noise level of a ship can be regarded as a one dimensional random variable that varies with frequency. Therefore, the statistical characteristics of the ship's radiated noise are to be used in this paper. For a one dimensional real random variable, the probability density function can describe the probability that the output of this random variable is near a certain point. The probability density function $f(x)$ has the following properties:

$$
f(x) \geq 0
$$

$$
\begin{gathered}
\int_{-\infty}^{+\infty} f(x) d x=1 \\
P(a<x \leq b)=\int_{a}^{b} f(x) d x
\end{gathered}
$$

$P$ represents the probability value of in the interval $[a, b\}$.

Select a large number of valid data samples of the same type of ship under the same conditions, and calculated the sound pressure spectrum level of each sample. The frequency range of analysis is $20 \mathrm{~Hz} \sim 4 \mathrm{kHz}$, and the frequency resolution is $1 \mathrm{~Hz}$. Mathematical statistics are used to obtain the probability density distribution corresponding to each frequency. Use the probability density function to generate random numbers that satisfy the determined probability distribution at each frequency, and use them as the spectral characteristics of the ships radiated noise at any time. Figure 1 
shows a set of ship radiated noise spectra that satisfy the probability distribution.

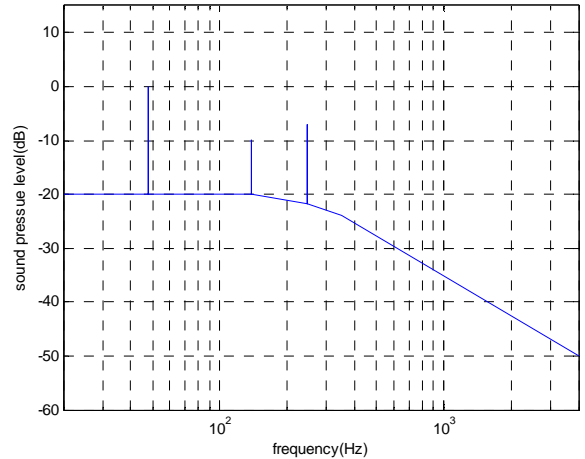

FIGURE I. RADIATED NOISE SPECTRUM SATISFYING PROBABILITY DISTRIBUTION

\section{WIDEBAND SIGNAL RECONSTRUCTION ALGORITHM BASED ON FOURIER SYNTHESIS}

Here, a wideband signal reconstruction algorithm based on Fourier synthesis is used for underwater acoustic signal waveform prediction. Broadband propagation was mainly used in the analysis of geo-acoustic propagation waveforms; the narrow band propagation model is mainly used in the analysis of underwater acoustics. With the increase of the sonar aperture, the common frequency is getting lower and lower, and the underwater acoustics field is also increasingly adopting the broadband technology mainly adopts the frequency domain Fourier synthesis method or the time domain method. Comparing with the complexity of the time domain solution, the Fourier synthesis technique is more practical, and it can more conveniently use the existing sound field numerical calculation method. The Fourier synthesis method performs the existing propagation model multiple times over several discrete frequencies in the frequency range at intervals of $\Delta f$, for example $\Delta f$ is $1 \mathrm{~Hz}$. Then, the propagation loss of each frequency in the obtained wide band is added by an appropriate weighted average process, and the propagation loss of the entire band is obtained.

Underwater acoustic propagation usually starts from the frequency domain wave equation (Helmholtz equation) and solves the sound pressure field at a certain frequency. If we study the problem of wave propagation of radiated noise from bulk acoustic sources in the ocean, we need to establish a broadband propagation model.

Based on the established single frequency acoustic propagation model, a broadband model was established using the Fourier synthesis method:

$$
p(r, z, t)=\frac{1}{2 \pi} \int_{-\omega_{\max }}^{\omega_{\max }} S\left(\omega_{k}\right) H\left(r, z, \omega_{k}\right) e^{-i \omega_{k} t} d \omega
$$

Here, $p(r, z, t)$ is the broadband signal waveform received at $t$ in the cylindrical coordinate system. $S\left(\omega_{k}\right)$ is the spectrum corresponding to the $\mathrm{k}^{\text {th }}$ frequency of the signal source, and its specific value is obtained from the statistical analysis in the previous section; $H\left(r, z, \omega_{k}\right)$ is the transfer function of the ocean channel.

According to ray acoustics, the total received signal is the superposition of all the signals arriving at the receiving point. The amplitude of the signal arriving along the $\mathrm{i}^{\text {th }}$ path is denoted as $A_{1}$, and the time delay of the signal arriving along the $i^{\text {th }}$ path is denoted as $\tau_{0 i}$. Therefore, the transmission function of the multi-path channel is:

$$
h(t)=\sum_{i=1}^{N} A_{i} \delta\left(t-\tau_{0 i}\right)
$$

The formula 5 means that the impulse response function of the channel is the waveform at the receiving point when the sound source emits a pulse. There are a total of $\mathrm{N}$ paths of sound ray that make an important contribution to the sound filed. Perform Fourier transform on both sides of equation 5 to get the following equation:

$$
H\left(r, z, \omega_{k}\right)=\sum_{i=1}^{N} A_{i} e^{-j 2 \pi f \tau_{0 i}}
$$

\section{DifFERENT CHANNEL MOdELS AND SHIP’s RADIATED NOISE MODELING}

$70 \%$ of the earth's surface is ocean. In the acoustic sense, as long as there are multiple contacts between the sound ray and the sea surface and the seabed, shallow sea conditions are considered. Therefore, some shallow seawater in the geographical sense belongs to the deep sea in the acoustic sense. Figure 2 left and right show typical sound speed profiles in shallow and deep seas, respectively. 

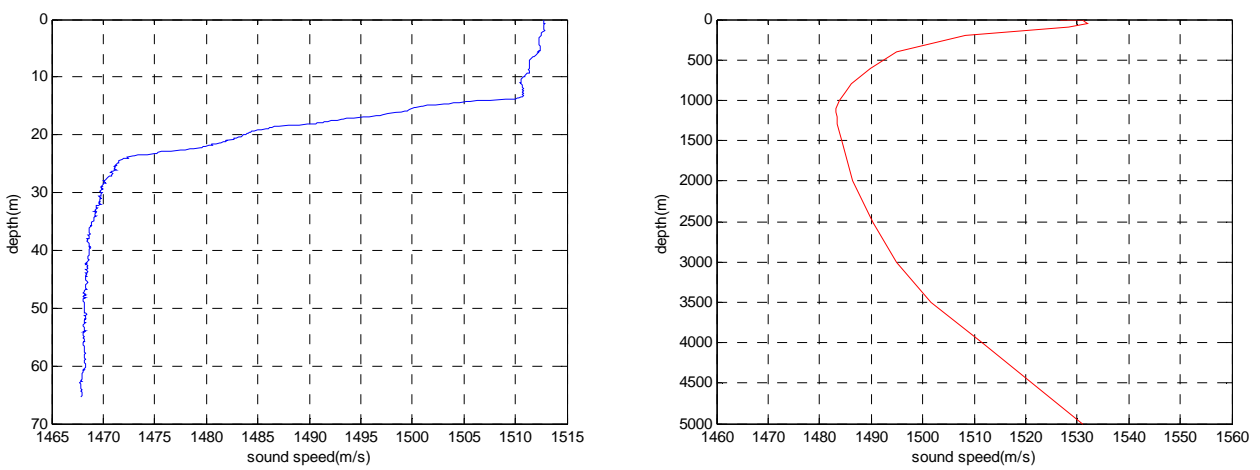

FIGURE II. SOUND SPEED PROFILE IN THE SHALLOW SEA AND IN THE DEEP SEA

Establish a horizontally uniform ocean waveguide environment, set acoustic parameters such as density, sound velocity, and attenuation coefficient of the seabed, and set the depth of the sound source and the depth of the receiver. Assuming that the ship sails at a constant speed on the sea, and figure 3 shows the horizontal distance between the hydrophone and the ship.

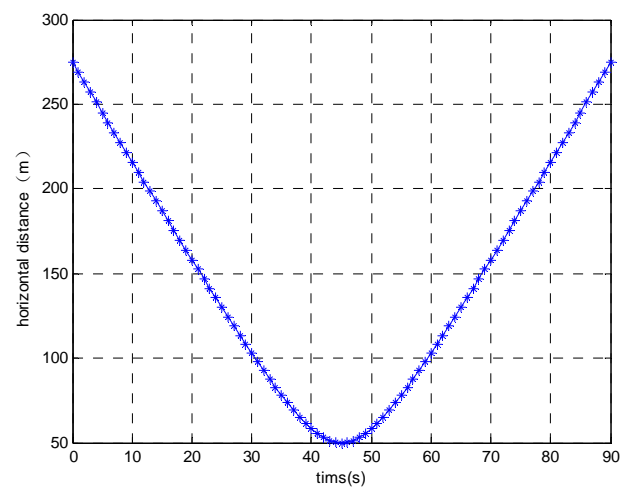

FIGURE III. HORIZONTAL DISTANCE BETWEEN THE HYDROPHONE AND THE SHIP OVER TIME

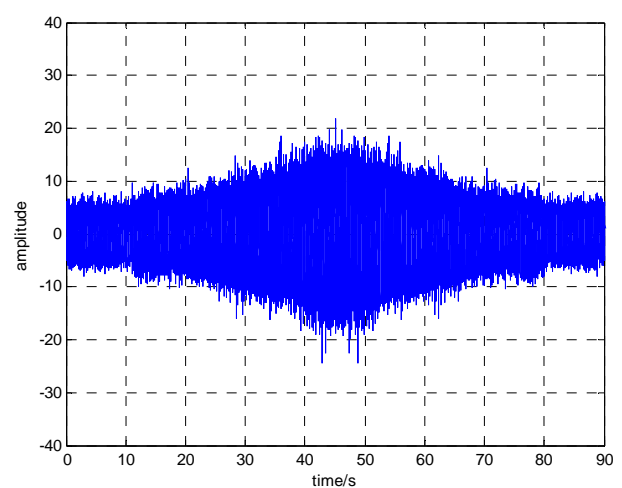

Using equation 6 and 4, the passage nose characteristics of the ship can be obtained. Figure 4 left figure shows the reconstructed results of the ship/s passing characteristics when the sound velocity in seawater is the sound speed profile shown in Figure 2 left. Figure 4 right figure shows the reconstructed results of the ship/s passing characteristics when the sound velocity in seawater is the sound speed profile shown in Figure 2 right figure. From the comparison between Fig.4 left and right, it can be seen that in the deep sea conditions, due to the effect of sound velocity profile of seawater on the trajectory of acoustic ray, the acoustic signal loss in the deep sea is relatively large. As a whole, the amplitude of the signal arriving at the receiving point in the shallow sea conditions slightly larger.

FIGURE IV. RECONSTRUCTED SIGNALS IN SHALLOW SEA AND IN DEEP SEA

\section{ANALysis OF THE EFFECTIVENESS OF RECONSTRUCTED WAVEFORMS}

A sample is randomly selected from the measured data

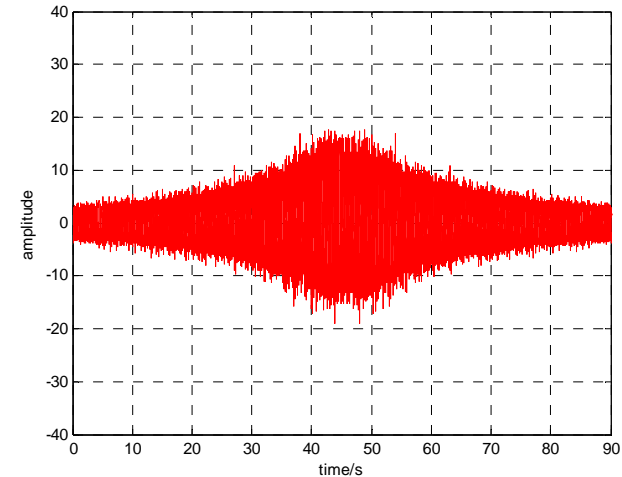

sample set for Fourier transform to obtain the spectrum of the measured radiated noise. In data analysis and feature modeling, it is often necessary to know the difference between the established model and the original features. This article uses 
correlation analysis to evaluate the effectiveness of the reconstructed signal. The specific plan is as follows: Perform Fourier transform on the time domain signal of the reconstructed ship's radiated noise to obtain the spectrum of the reconstructed signal.

The spectrum of the reconstructed signal is compared with the spectrum of the measured radiated noise signal. The definition of the correlation coefficient is as follows:

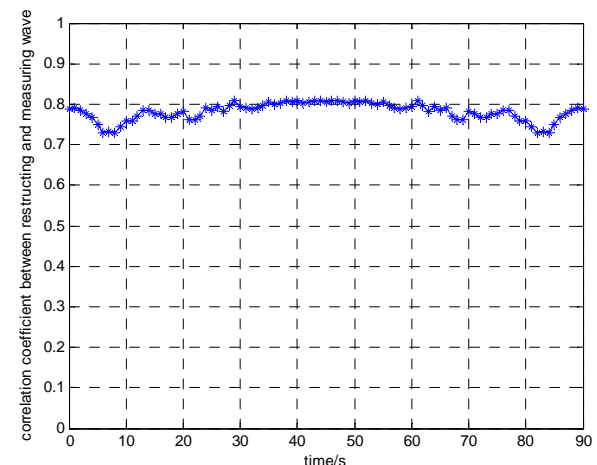

$$
R=\frac{\operatorname{Cov}\left(X(\omega)_{\mathrm{r}}, X(\omega)_{\mathrm{m}}\right)}{\sqrt{\operatorname{Var}\left(X(\omega)_{\mathrm{r}}\right) \operatorname{Var}\left(X(\omega)_{\mathrm{m}}\right)}}
$$

Here, $\operatorname{Cov}\left(X(\omega)_{\mathrm{r}}, X(\omega)_{\mathrm{m}}\right)$ is the covariance of reconstructed signal spectrum $X(\omega)_{\mathrm{r}}$ and measured signal spectrum $X(\omega)_{\mathrm{m}}, \operatorname{Var}\left(X(\omega)_{\mathrm{r}}\right)$ is variance of the reconstructed signal spectrum $X(\omega)_{\mathrm{r}}, \operatorname{Var}\left(X(\omega)_{\mathrm{m}}\right)$ is variance of the measured signal spectrum $X(\omega)_{\mathrm{m}}$.

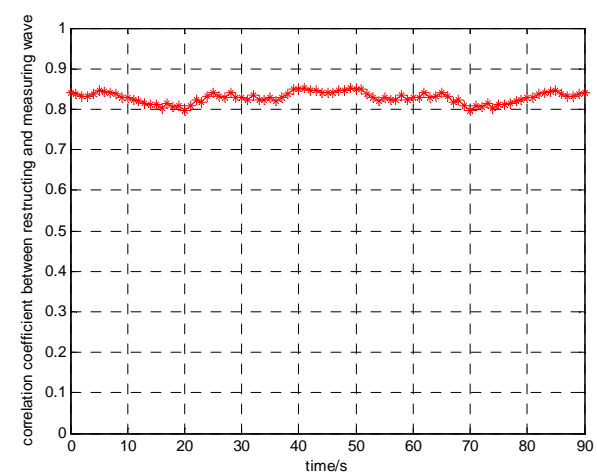

FIGURE V. SIMILARITY BETWEEN RECONSTRUCTED SIGNAL AND MEASURED SIGNAL IN SHALLOW SEA AND IN DEEP SEA

Figure 5 left and right show the correlation coefficient between reconstructed signal and measured signal in shallow sea and in the deep sea, respectively. It can be seen that there is a slight difference in frequency domain correlation at different distances for both shallow and deep sea water, but overall they are above 0.8 . Since the ship's radiated noise is a random signal, therefore, the time domain correlation between the reconstructed signal and the measured signal is poor.

\section{SUMMARY}

In this paper, we use a large number of near field measured ship's underwater radiated noise data to perform a statistical analysis of the characteristics of the spectrum, and to achieve a simulation of noise in different marine environments, and obtained the following conclusions:

1) Ship's radiated noise is a random signal. Even if the working conditions are invariable, the frequency and intensity of the line spectrum and the slope of the continuous spectrum also change. The statistical analysis method based on probability density distribution can effectively obtain the spectrum characteristics of ship's radiated noise.

2) The wideband Fourier synthesis algorithm has the advantage of fast calculation, and can simultaneously calculated the sound pressure values of multiple frequencies;

3) Using the modeling method of the ship's radiated noise passing characteristics proposed in this paper, the correlation between the received signal and the measured signal is greater than 0.8 , and the reliability of the reconstructed signal is higher;

4) The reconstructed passing characteristics can be used to study ship's noise characteristics under semi-physical conditions, and the engineering research of the model is being further developed.

\section{ACKNOWLEDGEMENT}

The authors would like to thank colleagues for assistance with data collection, and the organizers are thanked very much by the author for providing the international academic exchange opportunity.

\section{REFERENCES}

[1] Eugeniusz Kozaczka, Identification of hydro-acoustic waves emitted from floating units during mooring test, Polish Maritime Research.14 (2007)40-46.

[2] WANG Zhicheng, CHEN Zongqi, YU Feng, LIU Wenshuai et al.,Warship noise measuring and analyzing, , Publishing house of national defense industry, Bei Jing, 2004,pp.1-5.

[3] SHI Guangzhi, HU Junchuan. Study on target recognition based on simulation model of ship radiated noise, Journal of System Simulation. 18(2006) 1398-1401. 